\title{
BMJ Open Transcatheter closure, mini-invasive closure and open-heart surgical repair for treatment of perimembranous ventricular septal defects in children: a protocol for a network meta-analysis
}

\author{
Tao You, ${ }^{1}$ Kang Yi, ${ }^{1}$ Zhao-hong Ding, ${ }^{2}$ Xiao-dong Hou, ${ }^{1}$ Xing-guang Liu, ${ }^{1}$ \\ Xin-kuan Wang, ${ }^{1}$ Long $\mathrm{Ge}^{3}{ }^{3}$ Jin-hui Tian $^{3}$
}

To cite: You T, Yi K, Ding Z-hong et al. Transcatheter closure, mini-invasive closure and open-heart surgical repair for treatment of perimembranous ventricular septal defects in children: a protocol for a network meta-analysis. BMJ Open 2017;7:e015642. doi:10.1136/ bmjopen-2016-015642

- Prepublication history for this paper is available online. To view these files please visit the journal online (http://dx.doi. org/10.1136/bmjopen-2016015642).

Received 20 December 2016 Revised 10 April 2017 Accepted 17 May 2017

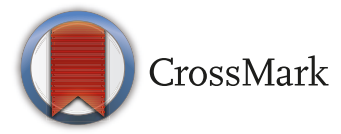

1Department of Cardiovascular Surgery, Gansu Provincial Hospital, Lanzhou, China ${ }^{2}$ Department of Nursing, Gansu Provincial Hospital, Lanzhou, China

${ }^{3}$ Evidence-Based Medicine Center, Lanzhou University, Lanzhou, China

Correspondence to Dr Tao You; youta02016@126.com

\section{ABSTRACT}

Introduction Both transcatheter device closure and surgical repair are effective treatments with excellent midterm outcomes for perimembranous ventricular septal defects (pmVSDs) in children. The mini-invasive periventricular device occlusion technique has become prevalent in research and application, but evidence is limited for the assessment of transcatheter closure, miniinvasive closure and open-heart surgical repair. This study comprehensively compares the efficacy, safety and costs of transcatheter closure, mini-invasive closure and openheart surgical repair for treatment of pmVSDs in children using Bayesian network meta-analysis.

Methods and analysis A systematic search will be performed using Chinese Biomedical Literature Database, China National Knowledge Infrastructure, PubMed, EMBASE.com and the Cochrane Central Register of Controlled Trials to include random controlled trials, prospective or retrospective cohort studies comparing the efficacy, safety and costs of transcatheter closure, miniinvasive closure and open-heart surgical repair. The risk of bias for the included prospective or retrospective cohort studies will be evaluated according to the risk of bias in non-randomised studies of interventions (ROBINS-I). For random controlled trials, we will use risk of bias tool from Cochrane Handbook version 5.1.0. A Bayesian network meta-analysis will be conducted using R-3.3.2 software. Ethics and dissemination Ethical approval and patient consent are not required since this study is a network meta-analysis based on published trials. The results of this network meta-analysis will be submitted to a peerreviewed journal for publication.

Protocol registration number CRD42016053352.

\section{INTRODUCTION}

Ventricular septal defects (VSDs) are the most common type of congenital heart disease, in which $80 \%$ are perimembranous ventricular septal defects (pmVSDs). ${ }^{1}$ Treatment of pmVSDs has been improved dramatically over the last 50 years. $^{2-4}$ Traditionally, open-heart surgical repair with midline sternotomy and
Strengths and limitations of this study

- To the best of our knowledge, this is the first network meta-analysis comparing the efficacy, safety and costs of transcatheter closure, miniinvasive closure and open-heart surgical repair for treatment of perimembranous ventricular septal defects in children.

- The results of this systematic review will help clinicians and patients to select appropriate repair methods.

- Our results will be limited by both the quantity and quality of the trials available for review.

cardiopulmonary bypass (CPB) has been the mainstay of therapy for many years; however, it is associated with morbidity, postoperative discomfort and a large thoracotomy scar. ${ }^{5}$ Catheter-based intervention was initially introduced for the closure of muscular VSDs and has been approved by the Food and Drug Administration in 2007. ${ }^{6}$ Transcatheter device closure of pmVSDs is a promising alternative $^{7-9}$ that has been widely used in developing countries, such as China and India, but it is not currently approved in the USA. $^{10}{ }^{11}$ Moreover, it remains a challenge for use on children with low body weight. ${ }^{1012}$ Previous pairwise meta-analysis suggests that there is no significant difference between transcatheter and surgical closure of pmVSDs in terms of early (up to 30 days) efficacy and safety in well-selected patients. ${ }^{13}$ During the same period, the mini-invasive periventricular device occlusion (MIPDO) technique, which combines the respective advantages of cardiac surgery, interventional cardiology and medical image techniques guided by transoesophageal echocardiography, became popular in research and application. ${ }^{14-17}$ 
Previously, there have been limited studies conducted that compare the efficacy between MIPDO, transcatheter and open-heart surgical closure for pmVSDs.

Network meta-analysis has become increasingly popular to evaluate healthcare interventions, since it allows to estimate the relative effectiveness among all interventions and rank ordering of the interventions. ${ }^{18}$ In the absence of head-to-head comparisons of all interventions of interest, indirect treatment comparison analyses using metwork meta-analyses of various randomised controlled trials (RCTs) can provide useful evidence to inform healthcare decision making. Even when the results of the direct comparisons are conclusive, combining them with indirect estimates in a mixed treatment comparison may yield more refined estimates. ${ }^{19} 20$

\section{OBJECTIVE}

The objectives of this study are to comprehensively compare the efficacy, safety and costs of transcatheter closure, mini-invasive closure and open-heart surgical repair for treatment of pmVSDs in children using Bayesian network meta-analysis.

\section{METHODS AND ANALYSIS \\ Design}

Bayesian network meta-analysis will be carried out in this study.

\section{Registration information}

We registered on the international prospective register of systematic review (PROSPERO) to publish our study protocol. The protocol of network meta-analysis is planed according to the preferred reporting items for systematic review and meta-analysis protocol (PRISMA-P) recommendation, and the PRISMA extension statement for reporting of systematic reviews incorporating network meta-analyses of healthcare interventions. ${ }^{21} 22$

\section{Information source}

A systematic search will be performed using Chinese Biomedical Literature Database, China National Knowledge Infrastructure, PubMed, EMBASE.com and the Cochrane Central Register of Controlled Trials. The references of included articles and relevant systematic reviews will be tracked to identify other relevant studies. The preliminary searches were performed on 19 December 2016.

\section{Search strategy}

Search terms will be: ventricular septal defect*, perimembranous, peri-membranous, VSD, occlusion, transcatheter, percutaneous, mini-invasive, sternotomy and child. Full details of the search strategy regarding PubMed are:

( ( ( (('Heart Septal Defects, Ventricular' [Mesh]) OR (('ventricular septal defect*'[Title/Abstract]OR VSD[Title/Abstract] $)))$ AND ((thorascopic[Title/
Abstract] OR sternotomy[Title/Abstract] OR 'minimally invasive' [Title/Abstract] OR mini-invasive[Title/ Abstract] OR 'surgical closure'[Title/Abstract] OR transcatheter[Title/Abstract] OR 'percutaneous occlusion'[Title/Abstract] $))$ AND ((infant[MeSH] OR child $[\mathrm{MeSH}]$ OR adolescent $[\mathrm{MeSH}]))))$ AND (( perimembranous OR peri-membranous))).

\section{Eligibility criteria}

Type of patients: children younger than 18 years of age with pmVSDs confirmed by clinical and transthoracic echocardiographic and scheduled for transcatheter closure, mini-invasive closure or open-heart surgical repair.

Type of designs: random controlled trials, prospective or retrospective cohort studies, systematic reviews or meta-analyses will be also included to track their references.

Type of interventions: transcatheter closure, mini-invasive closure and open-heart surgical repair.

Type of outcomes: procedural success rate, operative time (min), intensive care unit (ICU) stay (hours), hospital stay (days), total cost, any residual shunt after procedure (residual shunt was classified as small if the width was $\leq 2 \mathrm{~mm}$ and as significant if $\geq 3 \mathrm{~mm}$ ), ${ }^{23}$ major complications (such as thromboembolism, endocarditis, repeat operation, death due to the procedure, complete atrioventricular block requiring a permanent pacemaker, new-onset valvular regurgitation requiring surgical repair and device embolisation requiring surgical removal) and minor complications (such as wound complication requiring intervention, groin haematoma, device embolisation with transcatheter removal, cardiac arrhythmia, new or increased valvular regurgitation of 2 grades or less, haemolysis requiring only medication, pericardial/pleural effusion, pneumothorax, pneumopericardium and pneumoderma requiring chest tube or aspiration). ${ }^{23}$

Other criteria: we will include trials reported in the English and Chinese languages. There will be no limitations on year of publication and publication status.

\section{Study selections}

Literature search records will be imported into ENDNOTE X6 software. Two independent reviewers will examine the title and abstract of studies found in the search to identify related studies according to eligibility criteria. Thus, full-text versions of all potentially relevant studies will be obtained. Excluded trials and the reasons for their exclusion will be listed and examined by a third reviewer.

\section{Data items}

A standard data abstraction form will be created using Microsoft Excel 2013 (Microsoft, Redmond, WA, USA, www.microsoft.com) to collect data of interest. Two independent reviewers will extract following data and conflict will be resolved by discussion, including first author, year of publication, location, study design, study period, study 
arms, sample, mean age, mean body weight, gender, VSD size, type of surgery, method of surgical closure, device used, mean device size, CPB time, median follow-up and outcomes. We will consider the following factors as effect modifiers: mean age, type of study design, mean body weight, VSD size, device used, year of publication, length of follow-up and sample size.

\section{Risk of bias individual studies}

The risk of bias of included prospective or retrospective cohort studies will be evaluated according to the tool for assessing risk of bias in non-randomised studies of interventions (ROBINS-I) ${ }^{24}$ including bias due to confounding (preintervention), bias in selection of participants into the study (preintervention), bias in classification of interventions (at intervention), bias due to deviations from intended interventions (postintervention), bias due to missing data (postintervention), bias in measurement of outcomes (postintervention), bias in selection of the reported result (postintervention) and overall risk of bias. We will evaluate risk of bias as low, moderate, serious, critical risk of bias and no information.

The risk of bias tool from Cochrane Handbook version 5.1.0 will be also used if random controlled trials are included, which including method of random sequence generation (selection bias), allocation concealment (selection bias), blinding (performance bias and detection bias), incomplete outcome data (detection bias), selective reporting (detection bias) and other bias. ${ }^{25} \mathrm{We}$ will evaluate risk of bias as low, high or unclear risk of bias.

The risk of bias assessment will be completed by two independent reviewers, and conflicts will be resolved by a third reviewer.

\section{Geometry of the network}

A network plot will be drawn to describe and present the geometry of transcatheter closure, mini-invasive closure and open-heart surgical repair using R-3.3.2 software ( R Foundation for Statistical Computing, Vienna, Austria). Nodes will be used to represent different interventions and edges to represent the head-to-head comparisons between interventions. The size of nodes and thickness of edges are associated with sample sizes of intervention and numbers of included trials, respectively.

\section{Statistical analysis}

A Bayesian network meta-analysis will be performed using package 'gemtc' version 0.8.1 of R-3.3.2 software. ${ }^{26}$ The function mtc.run will be used to generate samples from using the Markov chains Monte Carlo sampler. Four Markov chains will be run simultaneously. We will set 5000 simulations for each chain as the 'burn-in' period. Then posterior summaries will be based on 50000 subsequent simulations. The model convergence will be assessed using Brooks-Gelman-Rubin plots method. ${ }^{27}$

\section{Summary measures}

Posterior medians of OR with $95 \%$ credible intervals (CrIs) will be used for procedural success rate, significant residual shunt, major complications and minor complications. Median mean differences or standard mean differences with $95 \%$ CrI for operative time, ICU stay, hospital stay and total cost. In addition, rank probabilities will be calculated, which indicate the probability for each treatment to be best, second best and so on. Clinical decisions about the choice of treatments can be recommended based on the results of rank probabilities when the differences in effect size of different treatments are small. ${ }^{28}$ The 'gemtc' package provides a matrix of the treatment rank probabilities as well as a plot of the rank probabilities.

\section{Analysis of heterogeneity}

We will assess clinical and methodological heterogeneity by carefully examining the characteristics and design of included trials. For pairwise meta-analysis, heterogeneity of treatment effects across head-to-head trials will be assessed by $\mathrm{I}^{2}$ statistics. If the $\mathrm{I}^{2}$ is $\leqq 50 \%$, it suggests that there is negligible statistical heterogeneity, and the fixed effects model will be used for meta-analysis. If the $\mathrm{I}^{2}$ is $>50 \%$, we will explore sources of heterogeneity by subgroup analysis and meta-regression using effect modifiers. If there is no clinical heterogeneity, the random effects model will be used to perform meta-analysis. In addition, we will also assess the global heterogeneity on the bias of the magnitude of heterogeneity variance parameter $\left(I^{2}\right.$ or $\tau^{2}$ estimated from the network meta-analysis models using the mtc.anohe command of the 'gemtc' package.

\section{Assessment of inconsistency}

If a loop connecting three arms exists, inconsistency between direct and indirect comparisons will be evaluated by a node splitting method. ${ }^{29}$

Funnel plot analysis

Publication bias will be examined with the Begg's ${ }^{30}$ and Egge's $^{31}$ funnel plot method. The comparison-adjusted funnel plot will be used to identify whether there will be a small sample effect between intervention networks.

\section{DISCUSSION}

Surgical repair through median sternotomy on CPB has been regarded as the gold method for treatment of pmVSDs. Hijazi et $a l^{2}$ first closed pmVSDs using an Amplatzer membranous VSD occlude in 2002. Over the past decade, some studies have found that the Amplatzer pmVSD occluder was associated with a relatively high risk of complete atrioventricular block. ${ }^{33}$ Interest has grown in the development of new techniques that can replace traditional open-heart surgery as the 'gold standard' for treatment of pmVSD. ${ }^{33}$ Recent RCTs demonstrated that both transcatheter device closure and surgical repair are effective treatments, with excellent midterm outcomes, for pmVSDs in children. ${ }^{33}$ The MIPDO technique combines the respective advantages of cardiac surgery, interventional cardiology and medical image techniques, 
and its use has become popular in research and application. ${ }^{14-17}$ To the best of our knowledge, there are no relevant RCTs to compare the differences of transcatheter closure, mini-invasive closure and open-heart surgical repair. The present study will first compare the efficacy, safety and costs of transcatheter closure, mini-invasive closure and open-heart surgical repair for treatment of pmVSDs in children using Bayesian network meta-analysis. However, some limitations are predictable. For example, costs are not reported in most studies, vary over time, different exchange rates and costs differences in different countries. In the USA, implants are performed by cardiologists, but in other countries, surgeons implant the devices, so surgical costs may be cheaper in some countries compared with device closure. Additionally, meta-analysis findings partially rely on the quality of original studies, and the number of eligible RCTs is predictably small.

\section{ETHICS AND DISSEMINATION}

\section{Ethical issues}

Ethical approval and patient consent are not required since this is a meta-analysis based on published studies.

\section{Publication plan}

This protocol has been registered on the international PROSPERO. ${ }^{34}$ The procedures of network meta-analysis will be conducted according to the PRISMA extension statement for reporting of systematic reviews incorporating network meta-analyses of healthcare interventions. The results of this network meta-analysis will be submitted to a peer-reviewed journal for publication.

\section{Acknowledgements The authors are grateful to MogoEdit for polishing and revising the language.}

Contributors Conception and design of research (TY, KY, ZD, XH and JT); tested the feasibility of the study (TY, KY, XL and XW); wrote the manuscript (TY); approved the final manuscript (TY, LG and JT).

\section{Competing interests None declared.}

Patient consent Patient consent are not required since this is a meta-analysis based on published studies.

Provenance and peer review Not commissioned; externally peer reviewed. Data sharing statement None.

Open Access This is an Open Access article distributed in accordance with the Creative Commons Attribution Non Commercial (CC BY-NC 4.0) license, which permits others to distribute, remix, adapt, build upon this work non-commercially, and license their derivative works on different terms, provided the original work is properly cited and the use is non-commercial. See: http://creativecommons.org/ licenses/by-nc/4.0/

(C) Article author(s) (or their employer(s) unless otherwise stated in the text of the article) 2017. All rights reserved. No commercial use is permitted unless otherwise expressly granted.

\section{REFERENCES}

1. Hoffman JI, Kaplan S. The incidence of congenital heart disease. J Am Coll Cardiol 2002;39:1890-900.

2. Sousa JE, Costa MA, Tuzcu EM, et al. New frontiers in interventional cardiology. Circulation 2005;111:671-81.
3. Fu YC, Bass J, Amin Z, et al. Transcatheter closure of perimembranous ventricular septal defects using the new Amplatzer membranous VSD occluder: results of the U.S. phase I trial. J Am Coll Cardiol 2006;47:319-25.

4. Quansheng X, Silin P, Zhongyun Z, et al. Minimally invasive perventricular device closure of an isolated perimembranous ventricular septal defect with a newly designed delivery system: preliminary experience. J Thorac Cardiovasc Surg 2009;137:556-9.

5. Rein JG, Freed MD, Norwood WI, et al. Early and late results of closure of ventricular septal defect in infancy. Ann Thorac Surg 1977;24:19-27.

6. Holzer R, Balzer D, Cao QL, et al. Device closure of muscular ventricular septal defects using the Amplatzer muscular ventricular septal defect occluder: immediate and mid-term results of a U.S. registry. J Am Coll Cardiol 2004;43:1257-63.

7. Butera G, Carminati M, Chessa M, et al. Transcatheter closure of perimembranous ventricular septal defects: early and long-term results. J Am Coll Cardiol 2007;50:1189-95.

8. Carminati M, Butera G, Chessa M, et al. Transcatheter closure of congenital ventricular septal defects: results of the european registry. Eur Heart $J$ 2007;28:2361-8.

9. Qin Y, Chen J, Zhao X, et al. Transcatheter closure of perimembranous ventricular septal defect using a modified doubledisk occluder. Am J Cardiol 2008;101:1781-6.

10. Gu M, You X, Zhao X, et al. Transcatheter device closure of intracristal ventricular septal defects. Am J Cardiol 2011;107:110-3.

11. Thanopoulos BV, Rigby ML, Karanasios E, et al. Transcatheter closure of perimembranous ventricular septal defects in infants and children using the Amplatzer perimembranous ventricular septal defect occluder. Am J Cardiol 2007;99:984-9.

12. Yang J, Yang L, Wan Y, et al. Transcatheter device closure of perimembranous ventricular septal defects: mid-term outcomes. Eur Heart J 2010;31:2238-45.

13. Saurav A, Kaushik M, Mahesh Alla V, et al. Comparison of percutaneous device closure versus surgical closure of perimembranous ventricular septal defects: A systematic review and meta-analysis. Catheter Cardiovasc Interv 2015;86:1048-56.

14. Zeng XJ, Sun SQ, Chen XF, et al. Device closure of perimembranous ventricular septal defects with a minimally invasive technique in 12 patients. Ann Thorac Surg 2008;85:192-4.

15. Quansheng X, Silin P, Zhongyun Z, et al. Minimally invasive perventricular device closure of an isolated perimembranous ventricular septal defect with a newly designed delivery system: preliminary experience. J Thorac Cardiovasc Surg 2009;137:556-9.

16. Zhang GC, Chen Q, Cao H, et al. Minimally invasive perventricular device closure of ventricular septal defect in infants under transthoracic echocardiograhic guidance: feasibility and comparison with transesophageal echocardiography. Cardiovasc Ultrasound 2013;11:8.

17. Xing $Q, P a n S, A n Q$, et al. Minimally invasive perventricular device closure of perimembranous ventricular septal defect without cardiopulmonary bypass: multicenter experience and mid-term follow-up. J Thorac Cardiovasc Surg 2010;139:1409-15.

18. Bafeta A, Trinquart $L$, Seror R, et al. Reporting of results from network meta-analyses: methodological systematic review. BMJ 2014;348:g1741.

19. Jansen JP, Schmid CH, Salanti G. Directed acyclic graphs can help understand Bias in indirect and mixed treatment comparisons. J Clin Epidemiol 2012:65:798-807.

20. Li L, Tian J, Tian H, et al. Network meta-analyses could be improved by searching more sources and by involving a librarian. $J$ Clin Epidemiol 2014;67:1001-7.

21. Moher D, Shamseer L, Clarke $M$, et al. Preferred reporting items for systematic review and meta-analysis protocols (PRISMA-P) 2015 statement. Syst Rev 2015;4:1.

22. Hutton B, Salanti G, Caldwell DM, et al. The PRISMA extension statement for reporting of systematic reviews incorporating network meta-analyses of health care interventions: checklist and explanations. Ann Intern Med 2015;162:777-84.

23. Chen ZY, Lin BR, Chen WH, et al. Percutaneous device occlusion and minimally invasive surgical repair for perimembranous ventricular septal defect. Ann Thorac Surg 2014;97:1400-6.

24. Sterne JA, Hernán MA, Reeves BC, et al. ROBINS-I: a tool for assessing risk of Bias in non-randomised studies of interventions. BMJ 2016;355:i4919.

25. Higgins JPT, Green S, The Cochrane Collaboration. Cochrance Handbook for Systematic Reviews of Interventions Version 5.1.0 [EB/ OL]. 2011. http://www.cochrane-handbook.org (16 May 2016).

26. van Valkenhoef G, Kuiper J. gemtc: Network meta-analysis using Bayesian methods. http://cran.rproject.org/web/packages/gemtcpdf (6 Dec 2016). 
27. Wu HY, Huang JW, Lin HJ, et al. Comparative effectiveness of renin-angiotensin system blockers and other antihypertensive drugs in patients with diabetes: systematic review and bayesian network meta-analysis. BMJ 2013;347:f6008.

28. Gelman A, Rubin DB. Inference from Iterative Simulation using multiple sequences. Statistical Science 1992;7:457-72.

29. Lu G, Ades AE. Combination of direct and indirect evidence in mixed treatment comparisons. Stat Med 2004;23:3105-24.

30. Begg CB, Mazumdar M. Operating characteristics of a rank correlation test for publication Bias. Biometrics 1994:50:1088-101.

31. Egger M, Davey Smith G, Schneider M, et al. Bias in meta-analysis detected by a simple, graphical test. BMJ 1997;315:629-34.
32. Hijazi ZM, Hakim F, Haweleh AA, et al. Catheter closure of perimembranous ventricular septal defects using the new Amplatzer membranous VSD occluder: initial clinical experience. Catheter Cardiovasc Interv 2002;56:508-15.

33. Yang J, Yang L, Yu S, et al. Transcatheter versus surgical closure of perimembranous ventricular septal defects in children: a randomized controlled trial. J Am Coll Cardiol 2014;63:1159-68.

34. You T, Yi K, Ding ZH, et al. Transcatheter closure, mini-invasive closure, and open-heart surgical repair for treatment of perimembranous ventricular septal defects in children: a network meta-analysis. PROSPERO 2016:CRD42016053352 http://www.crd. york.ac.uk/PROSPERO/display_record.asp?ID=CRD42016053352 